\title{
Orientation-contingent color aftereffects: Retinal specificity
}

\author{
LORRAINE G. ALLAN and C. A. G. HAYMAN \\ McMaster University, Hamilton, Ontario L8S 4K1, Canada
}

\begin{abstract}
Hayman and Allan (1980) concluded that the color aftereffects they obtained were orientation contingent rather than angle contingent, and they argued that these orientation-contingent color aftereffects were retinally specific. Additional data in support of the Hayman and Allan (1980) conclusion are reported. These data point to the important role of eye fixations and eye movements on contingent color aftereffects.
\end{abstract}

Murch (1969) reported that the McCollough effect extended over a test pattern twice the angular size of the adaptation patterns, and he concluded that the McCollough effect was not specific to the adapted portion of the retina. Stromeyer (1972) replicated this finding with uncontrolled fixation during test. With controlled fixation, the aftereffect decreased in saturation as the extent of overlap was reduced and completely disappeared when the test pattern was only about $.5 \mathrm{deg}$ off the adapted area. The finding reported by Harris (1969), that opposite aftereffects can be produced on adjacent areas of the retina, also suggests that the McCollough effect is retinally specific. Overall, the available evidence, although limited, suggests that orientation-contingent color aftereffects are specific to adapted portions of the retina.

In an earlier paper, we (Hayman \& Allan, 1980) concluded that the color aftereffects we obtained with ascending/descending arrow adaptation patterns were orientation contingent rather than angle contingent. We based our argument largely on the Harris (1969) report. Although this report is widely cited, neither the details of the experiment nor the data have been published. In the experiments reported in this note, we used a colorimeter and we obtained detailed verbal color reports. While the colorimeter data are in agreement with the results summarized by Harris, the verbal reports are somewhat more difficult to interpret. Our purpose is to describe and comment on these color reports.

\section{EXPERIMENT 1}

\section{Method \\ Subjects. Eight subjects, participating as part of a course requirement, were tested individually. \\ Apparatus. All patterns were square, subtended $7 \mathrm{deg}$ of \\ This research was supported by a National Sciences and Engineering Research Council of Canada grant to L. G. Allan. We would like to thank Lynn Tobin for her assistance in Experi- ment 1 . Requests for reprints should be sent to Lorraine G. Allan, Psychology Department, McMaster University, Hamilton, Ontario L8S 4K1, Canada.}

visual angle, and were made from Letratone No. LT107. The width of each line subtended $6.5 \mathrm{~min}$ of visual angle. Standard $35-\mathrm{mm}$ slide projectors were used to present the patterns.

The two adaptation patterns are shown in Figure 1. Each pattern was projected through an Eastman Kodak filter: a Wratten No. 53 filter for green and a Wratten No. 32 filter for magenta. Of the test patterns in Figure 2, only A1, B1, and B2 were used in this experiment, and there was no fixation point.

The strength of the color aftereffect was measured with the colorimeter described by Hayman and Allan (1980). If orientation-contingent color aftereffects are retinally specific, then, with fixation on the vertical midline, the top half of Pattern A1 should appear one color and the bottom half the opponent color. For the B patterns, one pair of diagonal quadrants should appear one color and the other pair the opponent color. Therefore, Pattern A1 was horizontally divided, with each half covered by an orthogonal polarizing filter, and the B patterns were divided into four quadrants with each pair of diagonal quadrants covered by an orthogonal polarizing filter. A rotatable disk, consisting of a matrix of orthogonally polarized complementary color filters (CC30G for green and CC20M for magenta), was mounted in front of the test projector lens. The relative amounts of green and magenta on the various sections of the projected test pattern could be adjusted by rotation of the disk.

Procedure. The session was divided into three phases: baseline, adaptation, and test. The baseline and test phases were conducted under indirect illumination by a $60-\mathrm{W}$ bulb; the adaptation phase occurred in the dark. In all phases, the subject was instructed to fixate the center of the pattern.

During baseline, four colorimeter readings, using the method of adjustment, were obtained for each test pattern. The subject

(a)

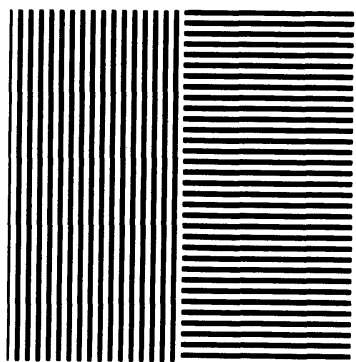

Figure 1. Adaptation patterns. Each pattern was square and subtended $7 \mathrm{deg}$ of visual angle. 
D1

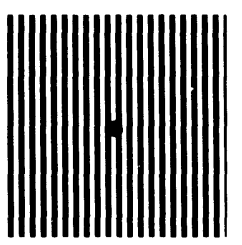

D2

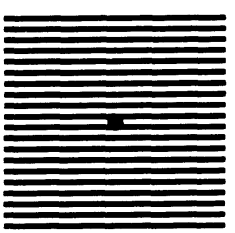

C1

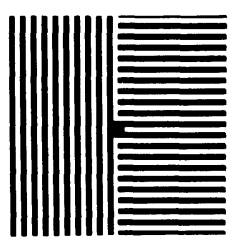

C2

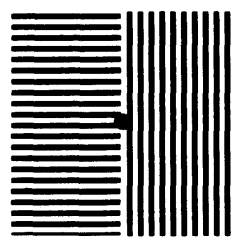

B1

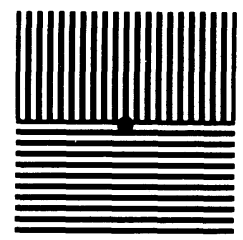

B2

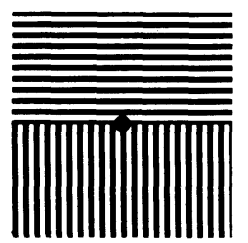

A1

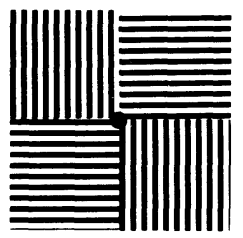

A2

Figure 2. Test patterns. Each pattern was square and subtended $7 \mathrm{deg}$ of visual angle. Patterns A1, B1, and B2 were used in Experiment 1. All the patterns were used in Experiment 2. The fixation point was not present in Experiment 1.

was instructed to rotate the disk until the pattern appeared colorless. All subjects were tested with Pattern A1; half the subjects were tested with B1 and the other half with B2. Presentation order of the test patterns was counterbalanced.

The adaptation phase was $15 \mathrm{~min}$. The two adaptation patterns were alternated every $5 \mathrm{sec}$. Two minutes separated adaptation from the test phase. Four colorimeter readings were again obtained for each test pattern. The colorimeter settings were followed by verbal reports about an unpolarized version of Pattern A1. The subject was asked whether he saw any colors, and if yes, what colors and at which locations.

\section{Results and Discussion}

Colorimeter settings. For each subject, the deviation of the mean of the four test colorimeter settings from the mean of the four baseline settings was determined. Of the eight subjects, seven gave appropriately signed deviation scores for the A1 pattern, and all subjects did so for the B patterns. Thus our colorimeter data are in agreement with the conclusion of Harris (1969) and Stromeyer (1972) that orientation-contingent color aftereffects are retinally specific.

Verbal reports. Given the consistent pattern of the colorimeter settings, we were surprised at the verbal reports that we obtained. No subject reported that the top half of the unpolarized A1 pattern was one color and the bottom half was the opponent color. Two subjects did report the expected color on the outer part of each quadrant, but with the opponent color on the inner part. In an attempt to understand the discrepancy between the colorimeter data and the verbal reports, more detailed verbal color reports, using a wider range of test patterns, were obtained in Experiment 2.

\section{EXPERIMENT 2}

\section{Method}

Subjects. Twelve paid subjects and the second author (C.A.G.H.) were tested individually. C.A.G.H. had considerable prior experience with contingent color aftereffects.
Apparatus. The adaptation patterns were those used in Experiment 1. The eight test patterns are shown in Figure 2. All the test patterns had a fixation point to aid in maintaining central fixation.

Procedure. Each experimental session consisted of two adaptation periods and two test periods. The first adaptation period was $20 \mathrm{~min}$. The two adaptation patterns were alternated every $5 \mathrm{sec}$. Two minutes separated adaptation from the test period. During the test period, the subject was asked to provide a detailed accurate description of four of the test patterns. The subject was then readapted for $2 \mathrm{~min}$. Again, $2 \mathrm{~min}$ separated adaptation from test. The subject gave detailed verbal color reports about the remaining four test patterns during the second test period.

Six subjects saw the test patterns in the order D1, C1, B1, $A 1, A 2, B 2, C 2$, and D2; six subjects saw the patterns in the reverse order.

\section{Results and Discussion}

Patterns D1 and D2. These patterns should be vertically split by two opponent colors. Of the 24 reports (12 subjects and two versions), 14 were appropriately located split color fields, 1 was an appropriately located color on one part of the pattern and no color on the other part, and two were appropriate colors on the outer parts of the pattern and no color or pale yellow on the central portion. There were five reports of no color anywhere on the pattern and one report of pale yellow. Either D1 or D2 was always presented first, and the six "no color"/"pale yellow" reports were given to that pattern. Only one report did not fit into any of the above categories.

Patterns B1 and B2. These patterns should appear as two diagonally located patches of one color and two diagonally located patches of the opponent color. There were 16 such reports and 4 additional reports of the appropriate color in at least two quadrants and no color in the remaining quadrants. Also, three reports indicated the appropriate color on the outer part of each quadrant and no color on the central portion. One report did not fit into any of the above categories. 
Patterns A1 and A2. The top half of these patterns should appear one color and the the bottom half the opponent color. There were only seven such reports. However, 10 reports located the appropriate colors on the outer parts of the pattern and the opponent color on the central portion. The remaining seven reports did not fall into the above categories and were quite variable.

Patterns C1 and C2. These patterns should appear one color. There were only two such reports. Ten reports indicated the appropriate color on the outer parts of the pattern and the opponent color on the central portion. Two reports indicated the appropriate color on one side of the pattern, the opponent color in the middle, and no color on the other side. Four reports indicated the appropriate color on one side and no color or pale yellow on the other side. Six of the reports did not fit into any of the above categories and were quite variable.

In summary, the verbal reports about the $\mathrm{A}$ and $\mathrm{C}$ patterns were considerably more variable than those about the B and D patterns. Thirty of the 48 verbal reports about the $B$ and $D$ patterns were in total agreement with that expected on the basis of retinal specificity. In addition, five reports localized the appropriate color on half the pattern with no color on the remaining part of the pattern and five reports localized the appropriate color on the outer parts of the pattern with no color on the central position. Thus, for B and D, 40 of the 48 reports are compatible with the retinal specificity hypothesis. In contrast, only 9 of the 48 reports about the $A$ and $C$ patterns appear to support the retinal specificity hypothesis. The most common report for the $\mathrm{A}$ and $\mathrm{C}$ patterns, 22 of the 48 reports, localized the expected colors on the outer parts of the pattern, but with opponent colors on the central portion.

A possible explanation for an opponent color on the central portion of the $\mathrm{A}$ and $\mathrm{C}$ patterns derives from a finding by Stromeyer $(1969,1971)$ that a contrast color can be induced by a color aftereffect. Small horizontal eye movements during adaptation would result in the center of the retina being stimulated by both green and magenta verticals and by both green and magenta horizontals, with the result that no contingent aftereffect would be acquired for this portion of the retina. It is possible that the spatially localized color aftereffects on the outer portions of the A and C patterns may have induced the opponent color on an achromatic central portion. One difficulty with this account is that central opponent colors were never reported on the B and D patterns. For these patterns, the aftereffects on each side are opponents, and therefore, each would induce a different color. No subject reported such a series of four alternating opponents. Thus, if we wish to assume that the opponent color reports for the $\mathrm{A}$ and $\mathrm{C}$ patterns are attributable to simultaneous color contrast, it becomes necessary to explain why a color induction pattern was never reported for the $B$ and $D$ patterns.

C.A.G.H. reported that he found it possible to manipulate the distribution of colors perceived on the $\mathrm{A}$ and $\mathrm{C}$ patterns. By maintaining an exact fixation at the center of the test pattern, $\mathrm{C}$ was seen as one color and $\mathrm{A}$ as two horizontal bands of opponent colors. However, C.A.G.H. found it difficult to achieve and hold this rigid fixation, and without prolonged central fixation, a vertical opponent color band was seen at the center. Interestingly, the opponent color arrangement on the $\mathrm{A}$ and $\mathrm{C}$ patterns was stable and more easily obtainable than the distribution requiring a rigid central fixation. C.A.G.H. did not perceive opponent colors on the central portions of the B and D patterns. Rather, lateral eye movements resulted in a displacement of the vertical boundary between the pink and green portions of the figure.

It is possible, then, that the opponent color on the central portion of the $\mathrm{A}$ and $\mathrm{C}$ patterns could be the result of small involuntary horizontal eye movements during test. Consider Figure 1a in magenta light and Figure $1 \mathrm{~b}$ in green light. With fixation on the vertical midline of $\mathrm{A} 1$, the top half should appear greenish and the bottom half pink. If the eyes move somewhat to the left of center, the top central verticals will appear pink and the bottom central horizontals greenish; if the eyes move somewhat to the right of center, the top central horizontals will appear pink and the bottom central verticals greenish. Thus, horizontal eye movements in either direction should result in the opponent color on the central portion of the A pattern. The same argument applies to the $\mathrm{C}$ pattern. For the $\mathrm{B}$ and $\mathrm{D}$ patterns, however, horizontal eye movements would not produce an opponent color; rather, the vertical boundary between the pink and greenish portions would shift with the eye movements.

It should be emphasized that the central opponent color pattern seen on the $\mathrm{A}$ and $\mathrm{C}$ patterns, while possibly the result of eye movements, is nevertheless a relatively stable percept. It is clearly the preferred percept and, according to C.A.G.H., the more "natural" percept. This stability, which requires temporal integration across eye "fixations," may be another example of the perceptual system's preference for a stable perceptual world (see Gregory, 1978).

\section{CONCLUDING COMMENTS}

The colorimeter data from Experiment 1 are in complete agreement with the report of Harris (1969) that lines of a particular orientation can produce an aftereffect of one color on one area of the retina while producing the opponent color on an adjacent area. The detailed verbal reports obtained in Experiment 2, while more "complex" than expected, can be taken to emphasize retinal specificity by identifying the important role of eye movements in contingent color aftereffects. A lateral eye movement will shift part of a test pattern from retina adapted to horizontal magenta to retina adapted to vertical green, with a resultant change in the perceived aftereffect.

It has been suggested to us that induction by simultaneous color contrast might account for the Hayman and Allan (1980) findings and that we were in error when we concluded that our aftereffects were orientation contingent rather than angle 


\section{ADAPTATION FIGURES}

(a)

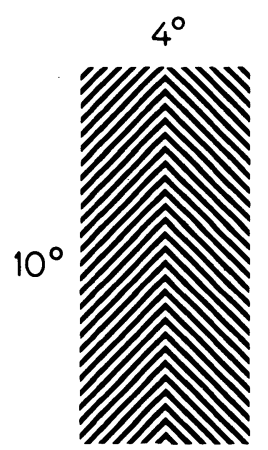

(b)

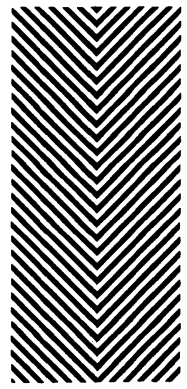

\section{TEST FIGURES}

(c)

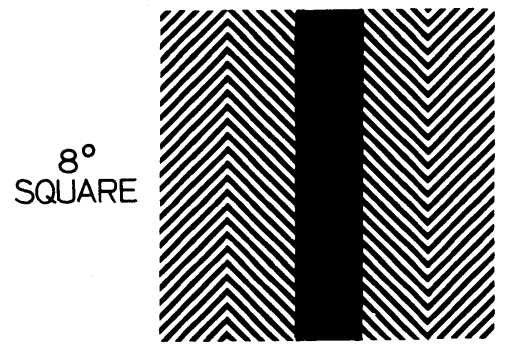

(d)

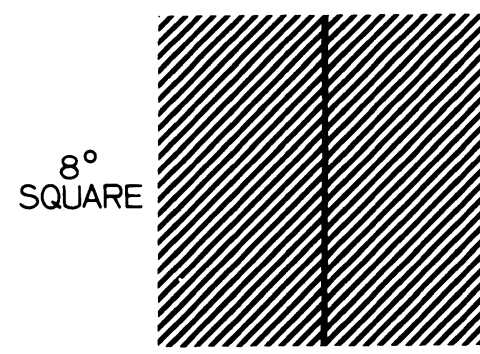

(e)

Figure 3. Adaptation and test patterns from Hayman and Allan (1980). The adaptation patterns subtended 4 deg of visual angle horizontally and $10 \mathrm{deg}$ vertically. The test patterns were square and subtended $8 \mathrm{deg}$.

contingent. If the aftereffects were angle contingent, then each individual arrow in Figure 3c should appear one color. However, our subjects reported that each arrow was vertically split by two opponent colors. Since the width of each adaptation figure was $4 \mathrm{deg}$ and the width of the test figure was $8 \mathrm{deg}$, it is conceivable that the color seen on the outer portion of each arrow was not an aftereffect but was induced by the aftereffect located on the inner portion. It should be noted, however, that the color reported for the inner portion was not the one expected for an angle-contingent aftereffect. Thus, even if the colors on the outer portion of each arrow were the result of induction, the aftereffect itself (the color on the inner portion of each arrow) was not contingent on angle. Moreover, the verbal reports for Figures $3 \mathrm{~d}$ and $3 \mathrm{e}$ provide no evidence for induction. Consider Figure $3 a$ in magenta light and Figure $3 b$ in green light. Figgure $3 \mathrm{~d}$ should appear vertically split, with green to the left of center and pink to the right. If induction of color by simultaneous contrast occurred, the green aftereffect would induce pink on the outer left part of the test pattern and the pink aftereffect would induce green on the outer right part. A series of four alternating opponent colors was never reported for this test pattern. Similarly, for Figure 3e, induction would predict a series of four alternating opponent colors for each horizontal band. Again, this was never reported.

The data from the experiments reported in this note provide a solid base for our earlier argument that aftereffects obtained using the ascending/descending arrow patterns are orientation contingent rather than angle contingent (Hayman \& Allan, 1980; White \& Riggs, 1974).

\section{REFERENCES}

Gregory, R. L., Eye and brain: The psychology of seeing. New York: McGraw-Hill, 1978.

HARRIS, C. S. Retinal localization of orientation-specific color aftereffects. Journal of the Optical Society of America, 1969, 59, 504. (Abstract)

Hayman, C. A. G., \& Allan, L. G. A reevaluation of anglecontingent color aftereffects. Perception \& Psychophysics, 1980, 28, 61-67.

Murch, G. M. Size judgments of McCollough afterimages. Journal of Experimental Psychology, 1969, 81, 44-48.

Stromeyer, C. F., III. Further studies of the McCollough effect. Perception \& Psychophysics, 1969, 6, 105-110.

Stromeyer, C. F., III. McCollough effect analogs of two-color projections. Vision Research, 1971, 11, 969-978.

Stromeyer, C. F., III. Contour-contingent color aftereffects: Retinal area specificity. American Journal of Psychology, 1972, 85, 227-235.

White, K. D., \& Riggs, L. A. Angle-contingent color aftereffects. Vision Research, 1974, 14, 1147-1154.

(Received for publication July 26, 1981.) 